\title{
COSMIC-RAY ENERGY CHANGES IN THE HELIOSPHERE. II. THE EFFECT ON K-CAPTURE ELECTRON SECONDARIES
}

\author{
R. A. Caballero-Lopez \\ Instituto de Geofísica, Universidad Nacional Autónoma de México, 04510, Mexico; rogelioc@geofisica.unam.mx \\ H. Moraal \\ School of Physics, North-West University, Potchefstroom 2520, South Africa \\ R. A. Mewaldt \\ Space Radiation Laboratory, California Institute of Technology, Pasadena, CA 91125 \\ F. B. McDonald \\ Institute for Physical Science and Technology, University of Maryland, College Park, MD 20742 \\ AND \\ M. E. WIEDENBECK \\ Jet Propulsion Laboratory, California Institute of Technology, Pasadena, CA 91109 \\ Received 2006 January 24; accepted 2007 March 22
}

\begin{abstract}
Recent accurate measurements of the cosmic-ray intensity ratio ${ }^{51} \mathrm{~V} /{ }^{51} \mathrm{Cr}$ below $1 \mathrm{GeV}$ nucleon ${ }^{-1}$ provide a powerful new tool to study cosmic-ray modulation in the heliosphere. This paper describes how energy changes during this modulation process influence this ratio. In particular, our model includes acceleration at the solar wind termination shock, and we find that this mechanism significantly enhances the ${ }^{51} \mathrm{~V} /{ }^{51} \mathrm{Cr}$ ratio at $1 \mathrm{AU}$. It is also shown that this acceleration makes the ratio more sensitive to the form of local low-energy interstellar spectra, below $\sim 100 \mathrm{MeV}$ nucleon $^{-1}$, than without it. Specifically, this acceleration provides an independent confirmation of the consensus that low-energy spectra should be flatter than their high-energy power-law forms.
\end{abstract}

Subject headings: cosmic rays - Sun: particle emission

\section{INTRODUCTION}

Adiabatic energy loss of cosmic rays in the radially expanding solar wind in the heliosphere is an integral part of cosmic-ray modulation, the other three processes being diffusion, convection, and drift in the solar wind and the heliospheric magnetic field (HMF). In a recent paper by Caballero-Lopez et al. (2006, hereafter Paper 1), it was shown that in a heliosphere that includes a solar wind termination shock (SWTS) and an extended heliosheath, the net energy loss may be significantly less than in standard models due to the fact that the shock accelerates particles, while in the near divergence-free solar wind in the heliosheath the intensity is modulated, with little or no energy loss.

Spectral shapes provide a powerful tool to study cosmic-ray properties, a good example being the difference between spectra of secondary and primary cosmic rays, which reflects the propagation, nuclear fragmentation, and escape of cosmic rays from the galaxy. Such processes are best studied at low energies, where the counting rates are high and good statistical accuracy can be achieved. However, at energies below $\approx 1 \mathrm{GeV}$ nucleon ${ }^{-1}$ these spectra are strongly modified by heliospheric modulation, with the mentioned adiabatic energy loss playing an important part. These modulation effects must first be unfolded before the shape of cosmic-ray spectra in interstellar space can be determined.

Another example where the relative abundance of cosmic-ray species provides a sensitive probe to study propagation effects is that of $\mathrm{K}$-capture electron cosmic-ray secondaries, as recently described by Niebur et al. (2003) and Mewaldt et al. (2004) using high-precision $A C E$ observations. Cosmic-ray nuclei attach electrons as they move through the galaxy. Typical attachment times are $10^{5} \mathrm{yr}$ for $10 \mathrm{MeV}$ nucleon $^{-1}$, and $10^{9} \mathrm{yr}$ for $10 \mathrm{GeV}$ nucleon $^{-1}$ nuclei. For a radioactive isotope such as ${ }^{51} \mathrm{Cr}$ that decays by electron capture, this attachment time is comparable with typical nuclear interaction and escape lifetimes at $T<100 \mathrm{MeV}$, and therefore it has a reasonable chance to occur. At higher energies, however, the attachment cross sections are smaller, and most of the ${ }^{51} \mathrm{Cr}$ fragments or escapes before this can happen. After this attachment, the nucleus decays into ${ }^{51} \mathrm{~V}$ with a half-life of 27 days, and consequently the ${ }^{51} \mathrm{~V} /{ }^{51} \mathrm{Cr}$ ratio in interstellar space should be strongly energy dependent, being much higher at low energies. According to current models $\sim 90 \%$ of ${ }^{51} \mathrm{Cr}$ and $>99 \%$ of ${ }^{51} \mathrm{~V}$ are of secondary origin, so that their relative abundance is governed by the fragmentation parameters (mainly from $\mathrm{Fe}$ ) and electron-capture decay, and not by the composition of the source material. Therefore, we study a secondary-to-secondary ratio. Niebur et al. (2003) and Mewaldt et al. (2004) have studied the energy dependence of this process, as modified by cosmic-ray modulation. They observed that the ${ }^{51} \mathrm{~V} /{ }^{51} \mathrm{Cr}$ ratio is less energy dependent at solar maximum conditions than at solar minimum. The authors interpreted this as a signature of different amounts of energy loss suffered by these particles, namely $\langle\Delta T\rangle \approx$ $200 \mathrm{MeV}$ nucleon $^{-1}$ at solar minimum and $\approx 400 \mathrm{MeV}$ nucleon $^{-1}$ at solar maximum. They used a solution of the one-dimensional (1D) cosmic-ray transport equation for their calculations. However, in Paper 1 it was shown that more complete solutions produce significantly different, mostly smaller, amounts of energy loss, and in this paper we therefore explore how different levels of sophistication of the modulation model affect this ratio. This will be done by accepting values of interstellar intensities and intensity ratios as input, without discussing their validity and inherent limitations. A complimentary approach can be found in Jones 


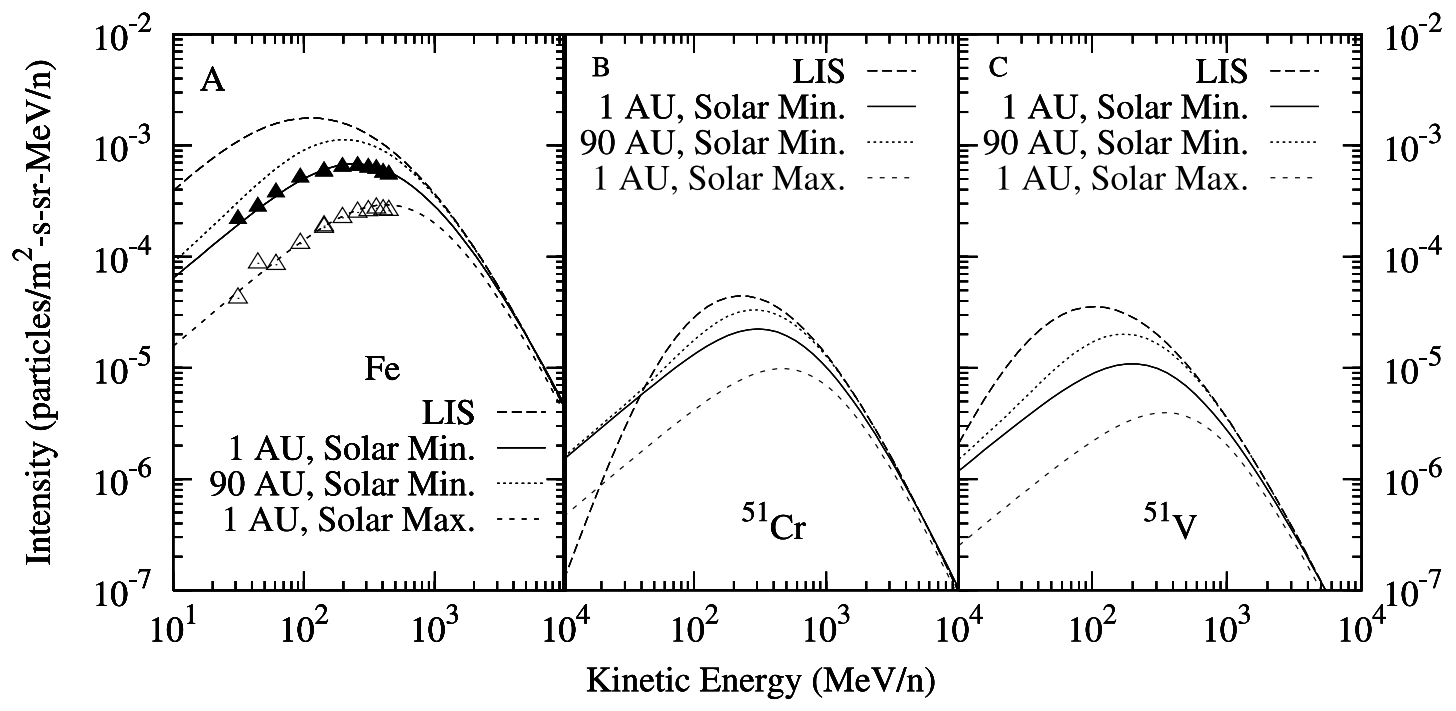

FIG. 1.- Local interstellar and modulated spectra of Fe, ${ }^{51} \mathrm{Cr}$, and ${ }^{51} \mathrm{~V}$. From top to bottom: LIS at $150 \mathrm{AU}$, together with modulated spectra at 90 AU (SWTS position) and at $1 \mathrm{AU}$, the latter at solar minimum and maximum. Fe spectra in panel $a$ also contain $A C E$ observations around solar minimum from 1997 August 28 to 1999 August 17 and around solar maximum from 2000 February 24 to 2003 January 5. The depletion of ${ }^{51} \mathrm{Cr}$ at low energies due to electron-capture decay is evident in its LIS.

et al. (2001), who reduced the entire heliospheric modulation process to a single force field parameter, but studied galactic propagation effects, with the emphasis on reacceleration of cosmic rays in the galaxy.

\section{CALCULATIONS}

We calculate ${ }^{51} \mathrm{~V} /{ }^{51} \mathrm{Cr}$ ratios in a variety of models for cosmicray modulation, ranging from (1) a simple force field solution of the transport equation; (2) a 1D (spherically-symmetric) numerical solution of the equation, such as used by Niebur et al. (2003) and Mewaldt et al. (2004); (3) a two-dimensional (2D) solution that also includes latitudinal diffusion and drifts; to (4) a solution that contains a SWTS at which particles are accelerated, plus a heliosheath in which the modulation occurs without energy loss. The transport equation and its solutions are described in detail in Caballero-Lopez et al. (2004) and in Paper 1, and the energy losses produced by the solutions are presented in Paper 1.

The modulation calculation starts with interstellar spectra from a model that fits the observed composition of cosmic rays with $4 \leq Z \leq 28$ (see, e.g., Yanasak et al. 2001). The solutions are done in a heliospheric model with an outer boundary at $r_{b}=$ $150 \mathrm{AU}$ and with a SWTS at $r_{s}=90 \mathrm{AU}$. Inside the shock, the solar wind velocity has the standard radial profile of $400 \mathrm{~km} \mathrm{~s}^{-1}$ in the ecliptic plane, increasing to $800 \mathrm{~km} \mathrm{~s}^{-1}$ between heliolatitudes $20^{\circ}$ and $30^{\circ}$ at solar minimum. During solar maximum we take $V=400 \mathrm{~km} \mathrm{~s}^{-1}$ at all latitudes. At the SWTS the wind speed drops by a factor of $s$, which is the compression ratio of the shock, and in the heliosheath it falls off further as $1 / r^{2}$. Unless otherwise stated, $s=3$ in this paper. The diffusion tensor $K(\boldsymbol{r}, P, t)$ contains elements $\kappa_{\|}(\boldsymbol{r}, P, t)$ and $\kappa_{\perp}(\boldsymbol{r}, P, t)$ for scattering along and perpendicular to the heliospheric magnetic field $(\mathrm{HMF}), \boldsymbol{B}$, together with an antisymmetric coefficient $\kappa_{T}=$ $\beta P /(3 B)$, which describes gradient, curvature, neutral sheet, and shock drift effects. The HMF structure is described by the standard Parker spiral magnetic field given in spherical polar coordinates $(r, \theta, \phi)$ by $\boldsymbol{B}=B_{e}\left(r_{e} / r\right)^{2}\left(\boldsymbol{e}_{r}-\tan \psi \boldsymbol{e}_{\phi}\right)$ with $\tan \psi=$ $\Omega\left(r-r_{0}\right) \sin \theta / V$, where $\Omega$ is the angular frequency of solar rotation, and $r_{0}$ is the Alfvén radius of several solar radii. The value of the field at Earth, $B_{e}$, at radius $r_{e}$, is taken as $5 \mathrm{nT}$ at solar minimum, and $10 \mathrm{nT}$ at solar maximum. The tilt angle of the neutral sheet is $10^{\circ}$ and $70^{\circ}$ at solar minimum and maximum, respectively. The effective radial and latitudinal diffusion coefficients are $\kappa_{r r}=$ $\kappa_{\|} \cos ^{2} \psi+\kappa_{\perp} \sin ^{2} \psi$ and $\kappa_{\theta \theta}=\kappa_{\perp}$, respectively. We choose $\kappa_{r r}=$ $1.2 \times 10^{23} \beta P(\mathrm{GV}) \mathrm{cm}^{2} \mathrm{~s}^{-1}$ inside the shock in the ecliptic plane, decreasing by a factor of 2 toward the poles, with $\kappa_{\theta \theta}=9.6 \times$ $10^{22} \beta P(\mathrm{GV}) \mathrm{cm}^{2} \mathrm{~s}^{-1}$, independent of latitude. At $P<0.4 \mathrm{GV}$ these coefficients continue $\propto \beta$. The solar maximum diffusion coefficients are 4.54 times smaller than the solar minimum values. In the heliosheath all diffusion coefficients are a factor $s$ smaller than inside the shock.

Figure 1 shows the basic solutions of the transport equation with these parameters for the spectra for $\mathrm{Fe},{ }^{51} \mathrm{Cr}$, and ${ }^{51} \mathrm{~V}$. Solutions are shown at 1 and $90 \mathrm{AU}$ at solar minimum conditions, and also at $1 \mathrm{AU}$ at solar maximum. Observations of Fe spectra are included to demonstrate that the parameters used provide a reasonable representation of solar minimum and solar maximum modulation. The upper dashed lines are the local interstellar spectra (LIS) taken from Mewaldt et al. (2004). These spectra have the form $j \propto P^{-2.35}$ at the source and are modified by propagation through the Galaxy. Notice that the 1 AU low-energy spectra have the classical $j \propto T$ form due to adiabatic energy losses. At 90 AU, however, the low-energy spectra have a steeper positive slope and form a "hump" because of shock acceleration and relatively little adiabatic energy loss. This is best seen on the ${ }^{51} \mathrm{Cr}$ and ${ }^{51} \mathrm{~V}$ spectra. Also notice from the ${ }^{51} \mathrm{Cr}$ spectra in Figure $1 b$ that even at $90 \mathrm{AU}$ (and also in the heliosheath at $r>$ $90 \mathrm{AU}$, but not shown) there are more adiabatically cooled particles at $T<30 \mathrm{MeV}$ nucleon $^{-1}$ than in the LIS, although there are no energy losses at $r \geq 90 \mathrm{AU}$. These adiabatically cooled particles are convected outward from deep inside the heliosphere.

Figure 2 shows the ${ }^{51} \mathrm{Cr} /{ }^{51} \mathrm{~V}$ ratios for these spectra. The line marked LIS is the ratio in interstellar space, which results from the two LISs in Figures $1 b$ and $1 c$. The data points are the $A C E$ observations taken from Niebur et al. (2003) for solar minimum conditions in Figure $2 a$ and for solar maximum in Figure $2 b$. Four modulated ratios are also shown in each panel. The dashed ratio marked "Shock" is produced at 1 AU by the full shock plus heliosheath solutions of Figures $1 b$ and $1 c$. The other three are for progressively simpler modulation models to demonstrate the significance of the various modulation effects. 


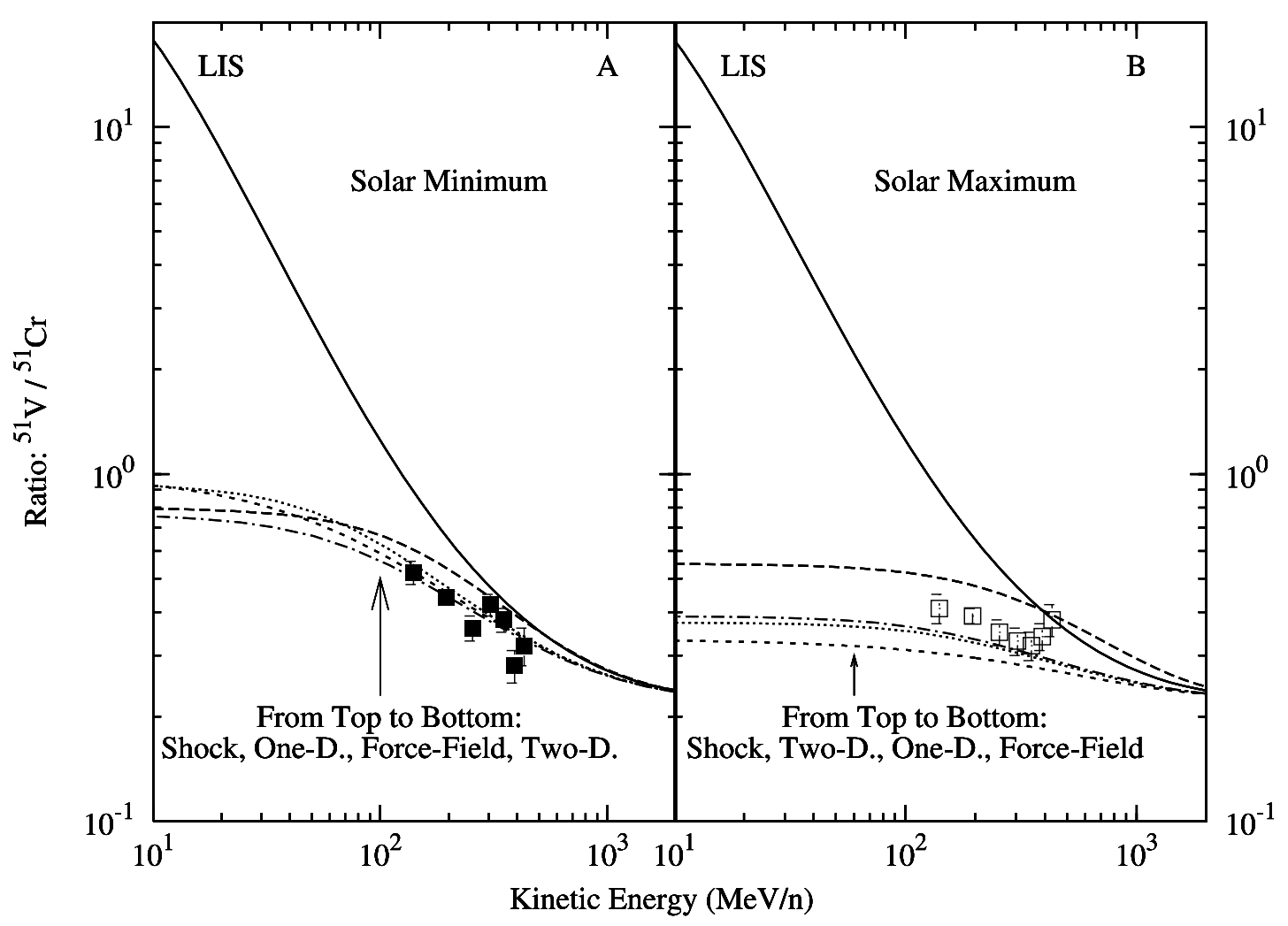

FIG. 2. $-{ }^{51} \mathrm{~V} /{ }^{51} \mathrm{Cr}$ ratio for $(a)$ solar minimum and $(b)$ solar maximum conditions. The modulated ratios are for 1 AU, and for four models of the modulation as

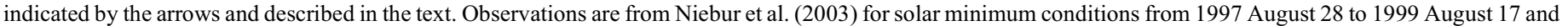
solar maximum conditions from 2000 February 24 to 2003 January 5.

First we note that all the low-energy modulated ratios are much lower than the interstellar value. The reason is due to adiabatic energy loss, because Figure $1 b$ shows that this process produces copious amounts of low-energy ${ }^{51} \mathrm{Cr}$ at $1 \mathrm{AU}$, even if there is very little at low energy in the LIS. Notice that at solar maximum the 1 AU ratio at $T>400 \mathrm{MeV}$ nucleon $^{-1}$ is higher than the LIS ratio. This is a shock acceleration effect: low-energy ${ }^{51} \mathrm{Cr}$ and ${ }^{51} \mathrm{~V}$ particles are accelerated up to these energies, but because there are fewer low-energy ${ }^{51} \mathrm{Cr}$ particles available at these low energies to be accelerated (compare Figs. $1 b$ and $1 c$ ), the ratio at high energies $\left(T>400 \mathrm{MeV}\right.$ nucleon $\left.^{-1}\right)$ increases.

The dash-dotted solution marked "Two-D" is for the same basic model but without a shock, which means that the supersonic solar wind continues unabated out to $r_{b}=150 \mathrm{AU}$. This, as well as the following two further approximations, is not meant to be a realistic model of the heliosphere. They are used to single out the main effects addressed in this paper, namely that of shock acceleration at the SWTS, combined with the zero-energy change modulation in the heliosheath. These two effects are contained in the full solution just described, but they are absent in the three approximations to follow.

It is evident that this change has a small effect at solar minimum, but it decreases the solar maximum ratio by $\approx 30 \%$. The ratio is now less energy dependent due to the fact that the additional adiabatic energy loss suffered in the region between 90 and $150 \mathrm{AU}$ "stretches out" the ratio more evenly toward low energies.

The dotted solution marked "One-D" is the one-dimensional (spherically-symmetric) solution of Caballero-Lopez \& Moraal (2004), also used by Niebur et al. (2003) and Mewaldt et al. (2004). It is effectively the previous $2 \mathrm{D}$ approximation, with the latitudinal dependence of the transport and the drift effects removed. In this case the entire modulation is determined by a single mod- ulation parameter, $M=\int V d r / \kappa_{r r}=750 / \beta P$ with $P$ in $\mathrm{MV}$, or the equivalent force field parameter $\phi=\int V d r / 3 \kappa_{1}=250 \mathrm{MV}$ at solar minimum (where $\kappa_{1}=1.2 \times 10^{23} \mathrm{~cm}^{2} \mathrm{~s}^{-1} \mathrm{MV}^{-1}$ is the spatial part of $\kappa_{r r}$ ). For solar maximum these values are 4.54 times larger. Finally, the curve marked "FF" is the solution of the force field approximation to the transport equation (Gleeson \& Urch 1973), using identical parameters as in the 1D solution.

The differences between these solutions at solar minimum are small, and the data points are not sufficiently accurate to discriminate between the models. This representation demonstrates that the most important modulation mechanisms are radially inward diffusion and outward convection, as described by the force field and 1D (spherically-symmetric) solutions. Latitudinal diffusion and drift effects only play a secondary modifying role. The only solution that differs significantly from the others is the full shock plus heliosheath solution at solar maximum. We note that this solution for $s=3$ lies above the measured ratios, and the no-shock solutions all lie below them. If the cross sections for secondary production during galactic propagation, which were used to derive the LISs, are accurate, this indicates that at least a weak shock is more consistent with the data than no shock.

This analysis confirms the earlier results of Niebur et al. (2003) and Mewaldt et al. (2004), that the ${ }^{51} \mathrm{~V} /{ }^{51} \mathrm{Cr}$ ratio is heavily modulated from its interstellar value, and it shows that it is not overly sensitive to the precise properties of the heliosphere that determine the heliospheric transport parameters. In $\S 3$ we show, however, that there is a strong sensitivity to the form of the LIS at low energies, $T<100 \mathrm{MeV}^{\text {nucleon }}{ }^{-1}$.

\section{SENSITIVITY TO THE LIS}

Ionization energy loss during Galactic propagation causes the ${ }^{51} \mathrm{Cr}$ LIS to peak at a few hundred $\mathrm{MeV}$ nucleon ${ }^{-1}$ and to 


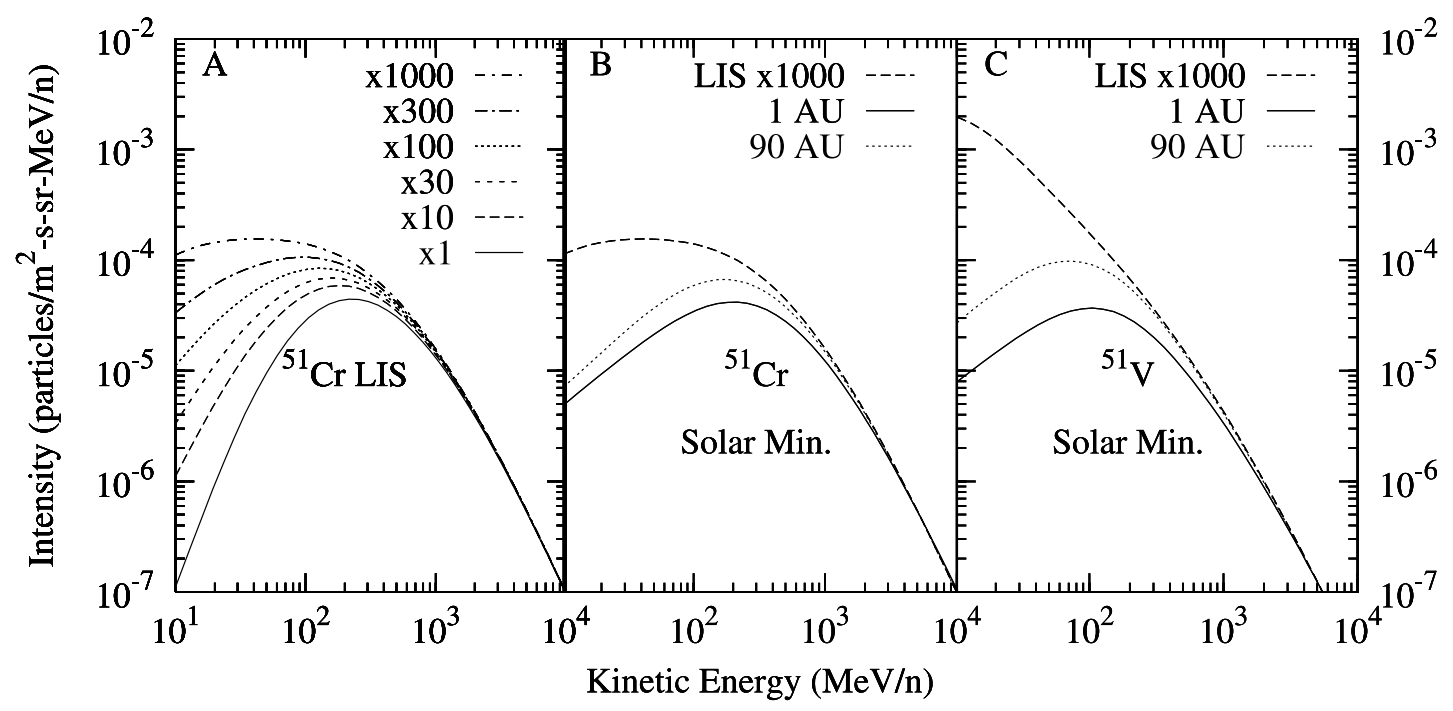

Fig. 3. - ${ }^{51} \mathrm{Cr}$ and ${ }^{51} \mathrm{~V}$ modulation with alternative LISs. In panel $a$ the ${ }^{51} \mathrm{Cr}$ LIS of Fig. $1 b$ is multiplied by $\exp \left[a /\left(T\left[\mathrm{MeV}\right.\right.\right.$ nucleon $\left.\left.\left.{ }^{-1}\right]+17\right)\right]$ with $a=52.2,91.9$, 124,134 , and 187 , to increase the LIS at $10 \mathrm{MeV}$ nucleon ${ }^{-1}$ by factors of $10,30,100,300$, and 1000 . The ${ }^{51} \mathrm{~V}$ LIS is multiplied by the same factors. Panels $b$ and $c$ show the modulated spectra at 1 and $90 \mathrm{AU}$ for solar minimum conditions, using the steepest of these input spectra.

decrease with decreasing energy below that. Figure $3 a$ shows a range of variations on this LIS, calculated by multiplying it with $\exp \left[a /\left(T\left[\mathrm{MeV}\right.\right.\right.$ nucleon $\left.\left.\left.^{-1}\right]+17\right)\right]$ with $a=52.2,91.9,124,134$, and 187 , which increases the intensity at $10 \mathrm{MeV}$ nucleon $^{-1}$ by factors of $10,30,100,300$, and 1000 . The ${ }^{51} \mathrm{~V}$ LIS is multiplied by the same factors. Figures $3 b$ and $3 c$ show the modulated spectra at 1 and $90 \mathrm{AU}$ for solar minimum conditions using the steepest of these input spectra (multiplication factor 1000). Due to adiabatic energy losses the modulated spectra at $1 \mathrm{AU}$ increase much less due to this variation than the LIS: at $1 \mathrm{AU}$ the ${ }^{51} \mathrm{Cr}$ and ${ }^{51} \mathrm{~V}$ spectra only increase with factors of $\sim 3$ and $\sim 8$, respectively. Nevertheless, the difference between these increases provides a probe for the form of their low-energy spectra in the Galaxy as follows.

The ${ }^{51} \mathrm{~V} /{ }^{51} \mathrm{Cr}$ ratio for these steeper input spectra are shown in Figure 4. The dashed lines in the three panels show the LIS ratio, which is not affected by this parameter variation, because the ${ }^{51} \mathrm{Cr}$ and ${ }^{51} \mathrm{~V}$ spectra are multiplied by the same factor. The solid lines in the three panels are the solution of the transport equation for solar minimum conditions at $1 \mathrm{AU}$ for the full shock plus heliosheath model. From top to bottom, these solutions are for high- to low-multiplication factors, as shown in Figure $4 b$. We note that the lowest solution of Figure $4 b$ is the same as the dashed solution in Figure $2 a$.

The overall message is that if there is no significant acceleration at the shock, as in Figure $4 a$, the ratio is insensitive to the form of the low-energy LIS, which a well-known consequence of adiabatic energy loss in the supersonic solar wind. However, when acceleration by the SWTS is effective, as in Figures $4 b$ and $4 c$, the modulated ${ }^{51} \mathrm{~V} /{ }^{51} \mathrm{Cr}$ ratio depends strongly on the form of the low-energy LIS of these species, and not just their ratio. The

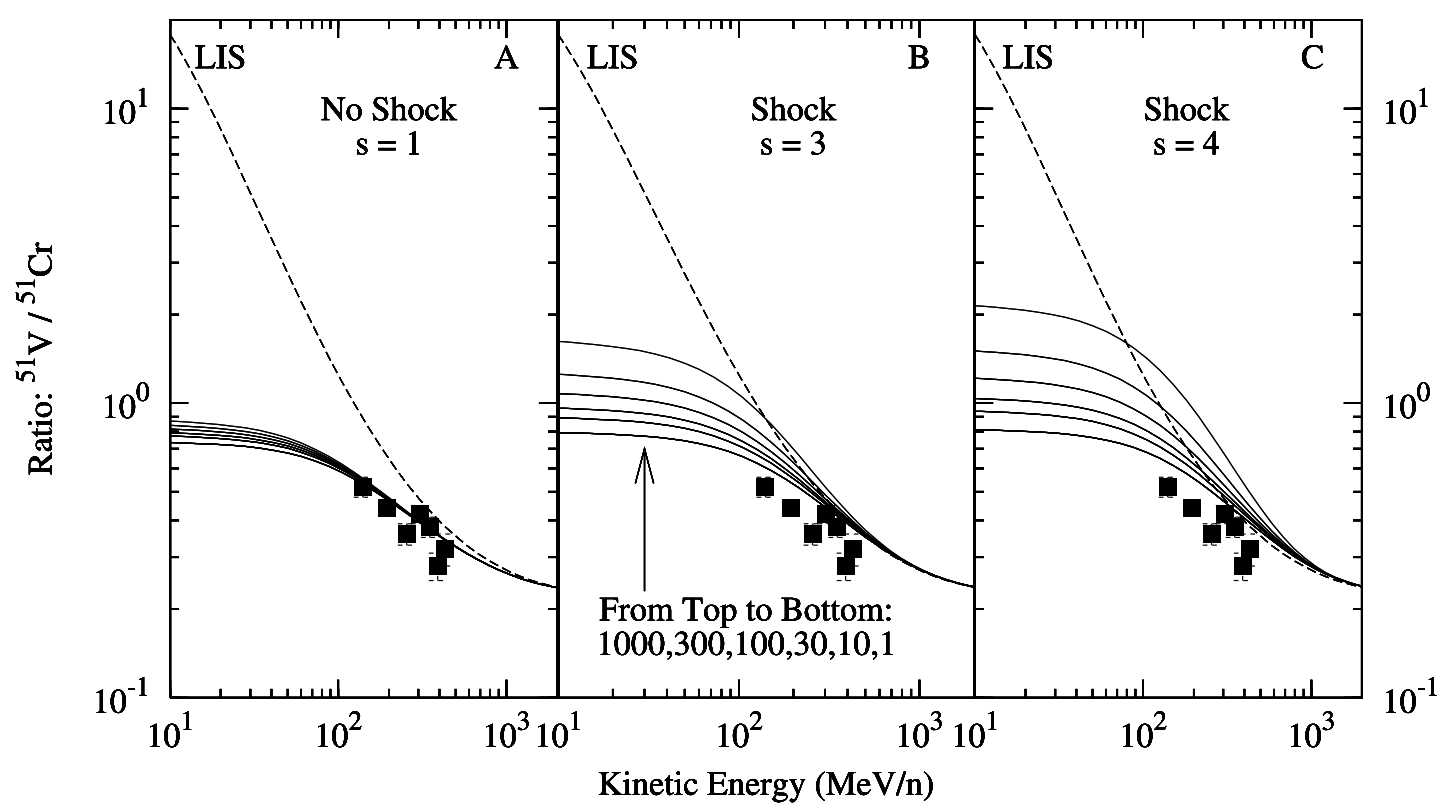

FIG. 4. - Same solar minimum ${ }^{51} \mathrm{~V} /{ }^{51} \mathrm{Cr}$ ratios as in Fig. 1, but for the alternative LISs of Fig. 3. All solid-line curves are for 1 AU. Only solutions for the full shock plus heliosheath model are shown, with three values of the compression ratio $s=1,3$, and 4 in panels $a, b$, and $c$, respectively. In all three panels the order of the solutions from top to bottom is for the multiplication factors as shown in the middle panel. 
reason lies in the difference in the spectral shapes of the two species. Since the ${ }^{51} \mathrm{~V}$ LIS is steeper than the ${ }^{51} \mathrm{Cr}$ one, there are more low-energy $\left(\sim 10 \mathrm{MeV} \text { nucleon }{ }^{-1}\right)^{51} \mathrm{~V}$ particles available for acceleration at the SWTS than ${ }^{51} \mathrm{Cr}$. This increases the ratio at middle energies $\left(\sim 100-300 \mathrm{MeV}\right.$ nucleon $\left.^{-1}\right)$ strongly. Thereafter, these accelerated particles lose their newly acquired energy adiabatically in the supersonic solar wind, increasing the ratio in the entire energy interval. The acceleration effect dies out naturally at $\sim 1 \mathrm{GeV}$ nucleon ${ }^{-1}$, because the diffusive length scale becomes larger than the shock dimensions, as described in, e.g., Steenberg \& Moraal (1999).

The best agreement with the observations is achieved with the no-shock solutions of Figure $4 a$, while the calculated ratios become progressively higher than the observed ones with increasing shock strength and steepness of the LIS. This provides an important diagnostic tool for the physics of cosmic-ray transport below $T<1000 \mathrm{MeV}$ nucleon $^{-1}$ at and beyond the SWTS: it seems that the observations favor interstellar spectra that are depleted at low energies as expected from ionization loss during galactic propagation (the bottom curves in each panel), and a shock that is a weak accelerator (Fig. $4 a$ ).

Stone et al. (2005) did not observe the simple shock-accelerated spectra expected for anomalous cosmic rays from a strong shock when Voyager 1 recently crossed the SWTS. This unexpected behavior is currently widely studied. Indications are that there may be alternative acceleration mechanisms, or that the source of the acceleration may be at different positions on the shock (McComas \& Schwadron 2006), or beyond the shock, as described in, e.g., Moraal et al. (2006). Florinski \& Zank (2006) explained the deviation from the expected shape as due to the dynamical effects of a merged interaction region (MIR). The limits set by the ${ }^{51} \mathrm{~V} /{ }^{51} \mathrm{Cr}$ ratio on this acceleration provide a useful new observational restriction for further acceleration studies. This method is more sensitive than modulation/acceleration studies with other species, because the nuclear physics determines that the LIS of a K-capture isotope, such as ${ }^{51} \mathrm{~V}$, is much steeper than that of those other species. We emphasize this point with a numerical example: the $100 \mathrm{MeV}^{51} \mathrm{~V} /{ }^{51} \mathrm{Cr}$ ratio at $1 \mathrm{AU}$ in Figure $4 b$ increases with a factor of $\approx 2$ from the bottom to the top curve, because the $100 \mathrm{MeV}^{51} \mathrm{Cr}$ intensity at $1 \mathrm{AU}$ increases with a factor of $\approx 2$ from Figures $1 b$ to $3 b$, but the ${ }^{51} \mathrm{~V}$ intensity increases with a factor of $\approx 4$.

We note that, not surprisingly, Jones et al. (2001) found that reacceleration of cosmic rays in the Galaxy may have a similar effect on the ${ }^{51} \mathrm{~V} /{ }^{51} \mathrm{Cr}$ ratio as that of SWTS acceleration found here.

\section{CONCLUSIONS}

Cosmic-ray electron capture secondaries offer an important probe for the physics of shock acceleration at the solar wind termination shock and modulation in the heliosheath, as well as for Galactic propagation processes that determine the form of lowenergy interstellar spectra. This augments an earlier conclusion by Caballero-Lopez et al. (2004) that observations at Earth are generally insensitive to modulation and acceleration conditions in the distant heliosphere. It was found there that the modulation integral $M=\int V d r / \kappa$, or equivalently the force field potential $\phi=1 / 3 \int V d r / \kappa_{1}$, is the most important parameter that determines the amount of modulation and spectral shape deep inside the heliosphere. It was stated that the values of these parameters are large enough at low energies so that adiabatic energy losses dominate and draw out the spectra into their classical $j \propto T$ shape, independent of the form of the LIS.

We have now demonstrated, however, that if low-energy ( $T \sim$ $10 \mathrm{MeV}$ nucleon $^{-1}$ ) intensities are sufficiently high, there are so many low-energy particles that get accelerated by the SWTS to energies between 100 and $1000 \mathrm{MeV}$ nucleon $^{-1}$, that this has a marked effect on spectra inside the heliosphere, and a ratio such as ${ }^{51} \mathrm{~V} /{ }^{51} \mathrm{Cr}$ will strongly increase in this energy region. The fact that such high ratios are not observed suggests that the interstellar spectra of cosmic-ray nuclei probably peak in the range of 100 $300 \mathrm{MeV}$ nucleon $^{-1}$, as expected from ionization losses suffered during their propagation through the galaxy. If this were not the case the SWTS would have to be a very weak cosmic-ray accelerator.

In view of the uncertainties in the acceleration observed by Voyager 1 during its crossing of the SWTS at 94 AU in 2004 December, such a weak acceleration cannot be ruled out. However, current studies, such as those of Schwadron \& McComas (2003), Stone et al. (2005), McComas \& Schwadron (2006), and Moraal et al. (2006) all preserve the basic underlying paradigm of acceleration in the outer heliosphere, but they invoke this acceleration at positions remote from Voyager 1 , such as at the poles, the ecliptic, the flanks, or other "favored acceleration locations" on the shock, and even beyond it, i.e., in the heliosheath. Observations like these at $1 \mathrm{AU}$ may provide important constraints to such refinements.

This work was supported by UNAM-DGAPA grant IN106105 in Mexico, NSF grant ATM 0107181 at Maryland, NASA grant NAG5-12929 at Caltech and JPL, and the South African National Research Foundation.

\section{REFERENCES}

Caballero-Lopez, R. A., \& Moraal, H. 2004, J. Geophys. Res., 109, A01101

Caballero-Lopez, R. A., Moraal, H., \& McDonald, F. B. 2004, J. Geophys. Res., 109, A05105

2006, Geophys. Res. Lett., 33, L12105 (Paper 1)

Florinski, V., \& Zank, G. P. 2006, Geophys. Res. Lett., 33, L15110

Gleeson, L. J., \& Urch, I. H. 1973, Ap\&SS, 25, 387

Jones, F. C., Lukasiak, A., Ptuskin, V. S., \& Webber, W. R. 2001, Adv. Space Res., 27, 737

McComas, D. J., \& Schwadron, N. A. 2006, Geophys. Res. Lett., 33, L04102

Mewaldt, R. A., et al. 2004, in AIP Conf. Proc. 719, Physics of the Outer Heliosphere, ed. V. Florinski, N. V. Pogorelov, \& G. P. Zank (New York: AIP), 127
Moraal, H., Caballero-Lopez, R. A., McCracken, K. G., McDonald, F. B. Mewaldt, R. A., Ptuskin, V. S., \& Wiedenbeck, M. E. 2006, in AIP Conf. Proc. 858, Physics of the Inner Heliosheath: Voyager Observations, Theory, and Future Prospects (New York: AIP), 219

Niebur, S. M., et al. 2003, J. Geophys. Res., 108, LIS 8-1

Schwadron, N. A., \& McComas, D. J. 2003, Geophys. Res. Lett., 30, 41-1

Steenberg, C. D., \& Moraal, H. 1999, J. Geophys. Res., 104, 24879

Stone, E. C., Cummings, A. C., McDonald, F. B., Heikkila, B. C., Lal, N., \&

Webber, W. R. 2005, Science, 309, 2017

Yanasak, N. E., et al. 2001, ApJ, 563, 768 